\title{
Electronic Journal of Graph Theory and Applications
}

\section{The Manhattan Product of Digraphs}

\author{
F. Comellas, C. Dalfó, M.A. Fiol \\ Departament de Matemàtica Aplicada IV, \\ Universitat Politècnica de Catalunya \\ Barcelona, Catalonia \\ $\{$ comellas,cdalfo,fiol\}@ma4.upc.edu
}

\begin{abstract}
We study the main properties of a new product of bipartite digraphs which we call Manhattan product. This product allows us to understand the subjacent product in the Manhattan street networks and can be used to built other networks with similar good properties. It is shown that if all the factors of such a product are (directed) cycles, then the digraph obtained is a Manhattan street network, a widely studied topology for modeling some interconnection networks. To this respect, it is proved that many properties of these networks, such as high symmetries, reduced diameter and the presence of Hamiltonian cycles, are shared by the Manhattan product of some digraphs. Moreover, we show that the Manhattan product of two Manhattan streets networks is also a Manhattan street network. Finally, some sufficient conditions for the Manhattan product of two Cayley digraphs to be also a Cayley digraph are given. Throughout our study we use some interesting recent concepts, such as the unilateral distance and related graph invariants.
\end{abstract}

Keywords: self-converse digraph, Manhattan street network, unilateral diameter, Cayley digraph, Hamiltonian cycle Mathematics Subject Classification: 05C20, 05C25, 05C12, 05C45, 90B10

\section{Introduction}

The 2-dimensional Manhattan street network $M_{2}$ was introduced simultaneously, in different contexts, by Morillo, Fiol and Fàbrega [13] and Maxemchuk [12] as an unidirectional regular mesh structure resembling locally the topology of the avenues and streets of Manhattan (or l'Eixample, in downtown Barcelona), see Fig. 3. In fact, $M_{2}$ has a natural embedding in the torus and it has been

Received: 17 October 2012, Accepted: 6 February 2013. 
extensively studied in the literature as a model of interconnection network. For instance, its average distance was computed by Khasnabish [11], and Chung and Agrawal [3], and the generation of routing schemes was studied by Maxemchuk [12]. Moreover, Chung and Agrawal [3] derived its diameter. Varvarigos [15] evaluated again the mean internodal distance, provided a shortest path routing algorithm and proved some Hamiltonian properties. In [4, 7], the authors formally defined the $n$-dimensional Manhattan street network $M_{n}$ and studied some of its structural properties. In particular, it was shown that $M_{n}$ is a Cayley digraph, which can be seen as a subgroup of the $n$ dimensional version of the wallpaper group pgg. The spectra of Manhattan street networks was also studied by the authors and Mitjana in [6].

Before outlining the contents of the paper, recall that a digraph $G=(V, A)$ consists of a set of vertices $V=V(G)$, together with a set of $\operatorname{arcs} A=A(G)$, which are ordered pairs of vertices, $A \subset V \times V=\{(u, v): u, v \in V\}$. An arc $(u, v)$ is usually depicted as an arrow with tail $u$ (initial vertex) and head $v$ (end vertex), that is, $u \rightarrow v$. The in-neighborhood $\Gamma_{G}^{-}(u)$ (respectively, out-neighborhood $\Gamma_{G}^{+}(u)$ ) of a vertex $u$ is the set of vertices adjacent to (respectively, from) $u$. The indegree of vertex $u$ is $\delta^{-}(u)=\left|\Gamma_{G}^{-}(u)\right|$, whereas its outdegree is $\delta^{+}(u)=\left|\Gamma_{G}^{+}(u)\right|$. Then, $G$ is $\delta$-regular when $\delta^{-}(u)=\delta^{+}(u)=\delta$ for every vertex $u \in V$. Given a digraph $G=(V, A)$, its converse digraph $\bar{G}=(V, \bar{A})$ is obtained from $G$ by reversing all the orientations of the arcs in $A$, that is, $(u, v) \in \bar{A}$ if and only if $(v, u) \in A$. A digraph $G$ is said to be self-converse when $\bar{G} \cong G$. In our study, the unilateral distance between two vertices $u, v \in V$ is defined to be

$$
\operatorname{dist}_{G}^{*}(u, v)=\min \left\{\operatorname{dist}_{G}(u, v), \operatorname{dist}_{G}(v, u)\right\}=\min \left\{\operatorname{dist}_{G}(u, v), \operatorname{dist}_{\bar{G}}(u, v)\right\},
$$

where $\operatorname{dist}_{G}$ is the standard (directional) distance for digraphs. From this concept, we also define the unilateral eccentricity $\varepsilon^{*}$ and the unilateral radius $r^{*}$ of a vertex $u$, and the unilateral diameter $D^{*}$ of $G$, as expected:

$$
\varepsilon^{*}(u)=\max _{v \in V}\left\{\operatorname{dist}_{G}^{*}(u, v)\right\}, r^{*}(G)=\min _{u \in V}\left\{\varepsilon^{*}(u)\right\}, \text { and } D^{*}(G)=\max _{u \in V}\left\{\varepsilon^{*}(u)\right\} .
$$

For instance, if $G=C_{N}$, the directed cycle on $N$ vertices, we have $r^{*}\left(C_{N}\right)=D^{*}\left(C_{N}\right)=\lfloor N / 2\rfloor$. Notice that all these parameters are obviously upper bounded by the corresponding parameters of the underlying graph (obtained from $G$ by changing arcs for edges). Some constructions of digraphs with large order for a given maximum degree and unilateral diameter were given by Gómez, Canale and Muñoz [8, 9].

Other standard definitions and basic results about graphs and digraphs not defined here can be found in Bang-Jensen and Gutin [1], Chartrand and Lesniak [2] and White [16]. Imrich and Klavzar gave a good description of the most common graph products in [10].

In this paper, we first recall the definition and some of the properties of the Manhattan street network (where the Manhattan product takes its name from). The motivation for this product is that it allows us to understand the subjacent product in the well-known Manhattan street networks and it can be used to build new networks with similar good properties. Afterwards, we define the Manhattan product of (self-converse bipartite) digraphs, which was introduced by the authors in [5]. It is shown that when all the factors are (directed) cycles, then the obtained digraph is just the Manhattan street network. Moreover, we prove that the Manhattan product of two Manhattan streets networks is also a Manhattan street network. It is also proved that many properties of these 
networks, such as high symmetries, reduced diameter and the presence of Hamiltonian cycles, are shared by the Manhattan product of some digraphs. Finally, we investigate when the Manhattan product of two Cayley digraphs is also a Cayley digraph and characterize the corresponding group.

\section{Manhattan street networks}

We recall the definition and some basic properties of a class of toroidal directed networks, commonly known as Manhattan street networks. For more information see $[4,7]$.

Given $n$ even positive integers $N_{1}, N_{2}, \ldots, N_{n}$, the $n$-dimensional Manhattan street network $M_{n}=M\left(N_{1}, N_{2}, \ldots, N_{n}\right)$ is a digraph with vertex set $V\left(M_{n}\right)=\mathbb{Z}_{N_{1}} \times \mathbb{Z}_{N_{2}} \times \cdots \times \mathbb{Z}_{N_{n}}$. Thus, each of its vertices is represented by an $n$-vector $\boldsymbol{u}=\left(u_{1}, u_{2}, \ldots, u_{n}\right)$, with $0 \leq u_{i} \leq N_{i}-1$, $i=1,2, \ldots, n$. The arc set $A\left(M_{n}\right)$ is defined by the following adjacencies (here called $i$-arcs):

$$
\left(u_{1}, \ldots, u_{i}, \ldots, u_{n}\right) \rightarrow\left(u_{1}, \ldots, u_{i}+(-1)^{\sum_{j \neq i} u_{j}}, \ldots, u_{n}\right), \quad i=1,2, \ldots, n .
$$

Therefore, $M_{n}$ is an $n$-regular digraph on $N=\prod_{i=1}^{n} N_{i}$ vertices.

The properties of $M_{n}$ are listed in the following result of the authors:

Theorem 2.1. [4] Let $M_{n}=M\left(N_{1}, N_{2}, \ldots, M_{n}\right)$ be an $n$-dimensional Manhattan street network. Then, the following statements hold:

(a) There exists an homomorphism from $M_{n}$ to the symmetric digraph of the hypercube $Q_{n}^{*}$, so that $M_{n}$ is a $2^{n}$-partite and bipartite digraph.

(b) The n-dimensional Manhattan street network $M_{n}$ is a vertex-symmetric digraph.

(c) For any $N_{1}, N_{2}$, the 2-dimensional Manhattan street network $M_{2}\left(N_{1}, N_{2}\right)$ is a line digraph.

(d) For $N_{i}>4, i=1,2, \ldots, n$, the diameter of an $n$-dimensional Manhattan street network $M_{n}=M\left(N_{1}, N_{2}, \ldots, N_{n}\right)$ is

$$
D= \begin{cases}\frac{N_{1}}{2}+\frac{N_{2}}{2}+1, & \text { if } N_{1} \equiv N_{2} \equiv 0 \bmod 4 \\ \frac{N_{1}}{2}+\frac{N_{2}}{2}, & \text { otherwise. }\end{cases}
$$

(e) The n-dimensional Manhattan street network $M_{n}$ is Hamiltonian.

\section{The Manhattan product and its basic properties}

In this section, we present an operation on (bipartite) digraphs which, as a particular case, gives rise to the Manhattan street networks. We begin with the more intuitive case of two factors. Let $G_{i}=\left(V_{i}, A_{i}\right)$ be two self-converse bipartite digraphs with independent vertex sets $V_{i 0}$ and $V_{i 1}$, $i=1,2$. Then, the definition of the Manhattan product $H=G_{1} \# G_{2}$ is as follows [5]: As usual, the vertex set of $H$ is the Cartesian (or direct) product $V_{1} \times V_{2}$. For the set of arcs, one starts with the direct product of $G_{1}$ and $G_{2}$, usually denoted by $G_{1} \square G_{2}$, and where there is an arc from $\left(u_{1}, u_{2}\right)$ to $\left(v_{1}, v_{2}\right)$ if either $u_{1}=v_{1}$ and $u_{2} \rightarrow v_{2}$ in $G_{2}$, or $u_{2}=v_{2}$ and $u_{1} \rightarrow v_{1}$ in $G_{1}$ 

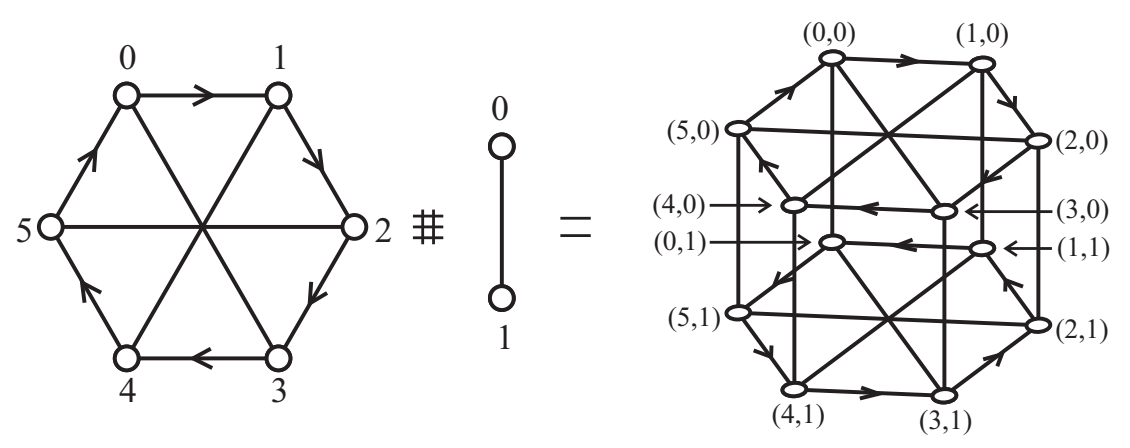

Figure 1. The Manhattan product Cay $\left(\mathbb{Z}_{6},\{1,3\}\right) \# K_{2}^{*}$ (undirected lines stand for pairs of arcs with opposite directions).

(see Fig. 4 for an example of a direct product). Then, one modifies this by changing some of the arrow directions according to the following rule: Because $G_{1}$ and $G_{2}$ are bipartite, each vertex of each graph comes with a prescribed type which is either 0 or 1 depending on which independent set, $V_{i 0}$ or $V_{i 1}$, it belongs to (since each $G_{i}$ is self-converse, it does not matter which partite set is $V_{i 0}$ or $\left.V_{i 1}\right)$. Then, an edge from $\left(w, u_{2}\right)$ to $\left(w, v_{2}\right)$ preserves its direction if $w$ is of type 0 , that is $w \in V_{10}$, and reverses its direction if it is of type $1, w \in V_{11}$. Similarly, an edge from $\left(u_{1}, w\right)$ to $\left(u_{2}, w\right)$ preserves its direction if $w \in V_{20}$ and reverses it if $w \in V_{21}$. Fig. 1 shows an example of the Manhattan product of the circulant digraph on six vertices and steps 1 and 3 (in other words, the Cayley digraph on $\mathbb{Z}_{6}$ with generating set $\{1,3\}$ ) by the symmetric complete digraph on two vertices, $K_{2}^{*}=\operatorname{Cay}\left(\mathbb{Z}_{2},\{1\}\right)$. One can, then, define the product of more bipartite digraphs by iterating and checking that the product is both associative and commutative. This lead us to the following more formal definition.

Definition 3.1. Let $G_{i}=\left(V_{i}, A_{i}\right)$ be $n$ bipartite self-converse digraphs with independent sets $V_{i}=V_{i 0} \cup V_{i 1}, N_{i}=\left|V_{i}\right|, i=1,2, \ldots, n$. Let $\pi$ be the characteristic function of $V_{i 1} \subset V_{i}$ for any $i$, that is, for $i=1,2, \ldots, n$,

$$
\pi(u):= \begin{cases}0 & \text { if } u \in V_{i 0}, \\ 1 & \text { if } u \in V_{i 1} .\end{cases}
$$

Then, the Manhattan product $H_{n}=G_{1} \# G_{2} \# \cdots \# G_{n}$ is the digraph with vertex set $V\left(H_{n}\right)=$ $V_{1} \times V_{2} \times \cdots \times V_{n}$, and adjacencies

$$
\left(u_{1}, \ldots, u_{i}, \ldots, u_{n}\right) \quad \rightarrow \quad\left(u_{1}, \ldots, v_{i}, \ldots, u_{n}\right), \quad i=1,2, \ldots, n,
$$

where

$$
v_{i} \in \Gamma^{\sigma}\left(u_{i}\right):= \begin{cases}\Gamma_{G_{i}}^{+}\left(u_{i}\right) & \text { if } \sigma=\sum_{j \neq i} \pi\left(u_{j}\right) \text { is even, } \\ \Gamma_{G_{i}}^{-}\left(u_{i}\right) & \text { if } \sigma=\sum_{j \neq i} \pi\left(u_{j}\right) \text { is odd. }\end{cases}
$$

Thus, if every $G_{i}$ is $\delta_{i}$-regular for $i=1,2, \ldots, n$, then $H_{n}$ is a $\delta$-regular digraph with degree $\delta=\sum_{i=1}^{n} \delta_{i}$ and $N=\prod_{i=1}^{n} N_{i}$ vertices. 
Notice that the same definition applies for non-necessarily self-converse digraphs, but then the product obtained depends on the characteristic function $\pi$ for each factor graph which, in turn, depends on the choice of 'colors' 0 and 1 for the independent sets of the corresponding factor graph. Then, in this case, one can obtain up to $2^{n}$ non-isomorphic products. By the way of example, take a digraph $G$ having three vertices $u, v, w$ and two $\operatorname{arcs} u \rightarrow v, w \rightarrow v$, as shown in Fig. 2. When taking the product $G \# G$, the four arcs incident with vertex $(v, v)$ may all be outgoing, $(G \# G)_{1}$, all incoming, $(G \# G)_{2}$, or two may be outgoing and two incoming, $(G \# \mid G)_{3}$, depending on the $0-1$ coloring of each of the two factors. In this particular case, the two factors are isomorphic, $G_{1}=G_{2}=G$, and we only get three non-isomorphic product digraphs and not four. Perhaps it would be interesting if one could find examples of factors that are not all self-converse but their Manhattan product is unique.

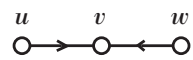

G

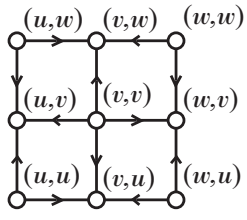

$(G \#)_{1}$

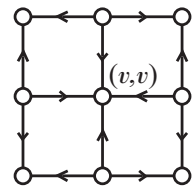

$(G \# G)_{2}$

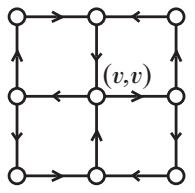

$(G \# G)_{3}$

Figure 2. Three non-isomorphic Manhattan products.

In our case of self-converse factors, some of the basic properties of their Manhattan product, which are a generalization of the properties of the Manhattan street networks given in [4, 7], are presented in the next proposition:

Proposition 3.1. The Manhattan product $H_{n}=G_{1} \# G_{2} \# \ldots \# G_{n}$ satisfies the following properties:

(a) $H_{n}$ is a bipartite self-converse digraph.

(b) There exists an homomorphism from $H_{n}$ to the symmetric digraph of the hypercube $Q_{n}^{*}$. Therefore, $H_{n}$ is a bipartite and $2^{n}$-partite digraph.

(c) Given $k=2,3 \ldots, n-1$ and any $n-k$ fixed vertices $x_{i} \in V_{i}, i=k+1, k+2, \ldots, n$, the subdigraph of $H$ induced by the vertex set

$$
\left\{\left(u_{1}, \ldots, u_{k}, x_{k+1}, \ldots, x_{n}\right): u_{1} \in V_{1}, \ldots, u_{k} \in V_{k}\right\}
$$

is isomorphic to the Manhattan product $H_{k}=G_{1} \# G_{2} \# \cdots \# G_{k}$. 
Proof. We only prove the properties $(b)$ and $(a)$ because $(c)$ can be proved similarly.

(b) The homomorphism from $H$ to $Q_{n}^{*}$ is

$$
\left(u_{1}, u_{2}, \ldots, u_{n}\right) \mapsto\left(\pi\left(u_{1}\right), \pi\left(u_{2}\right), \ldots, \pi\left(u_{n}\right)\right),
$$

which transforms each vertex of $H$ in a binary $n$-string as its image vertex in $Q_{n}^{*}$.

(a) As the Manhattan product is associative, we only need to deal with the case $H=G_{1} \# G_{2}$. For a given mapping $\phi$ and a vertex set $U$, let $\phi(U)$ denote the set $\{\phi(u): u \in U\}$. Since $G_{i} \cong \bar{G}_{i}$ for $i=1,2$, there exist isomorphisms $\psi_{i}$, such that $\psi_{i}\left(\Gamma_{G_{i}}^{\mp}\left(u_{i}\right)\right)=\Gamma_{G_{i}}^{ \pm}\left(\psi_{i}\left(u_{i}\right)\right)$ for all $u_{i} \in V_{i}$. As $\psi_{i}$ is a mapping between stable sets, the parity $\pi$ in $\bar{G}_{i}$ can be defined in such a way that $\pi\left(u_{i}\right)$ is even if and only if $\pi\left(\psi_{i}\left(u_{i}\right)\right)$ is also even. Then, the mapping $\Psi$ defined in $H$ as $\Psi\left(u_{1}, u_{2}\right):=\left(\psi_{1}\left(u_{1}\right), \psi_{2}\left(u_{2}\right)\right)$ is the automorphism from $H$ to its converse $\bar{H}$. Indeed,

$$
\begin{aligned}
\Psi\left(\Gamma_{H}^{+}\left(u_{1}, u_{2}\right)\right) & =\Psi\left(\left(\Gamma_{G_{1}}^{\pi\left(u_{2}\right)}\left(u_{1}\right), u_{2}\right)\right) \cup \Psi\left(\left(u_{1}, \Gamma_{G_{2}}^{\pi\left(u_{1}\right)}\left(u_{2}\right)\right)\right) \\
& =\left(\psi_{1}\left(\Gamma_{G_{1}}^{\pi\left(u_{2}\right)}\left(u_{1}\right)\right), \psi_{2}\left(u_{2}\right)\right) \cup\left(\psi_{1}\left(u_{1}\right), \psi_{2}\left(\Gamma_{G_{2}}^{\pi\left(u_{1}\right)}\left(u_{2}\right)\right)\right) \\
& =\left(\Gamma_{G_{1}}^{\pi\left(u_{2}\right)+1}\left(\psi_{1}\left(u_{1}\right)\right), \psi_{2}\left(u_{2}\right)\right) \cup\left(\psi_{1}\left(u_{1}\right), \Gamma_{G_{2}}^{\pi\left(u_{1}\right)+1}\left(\psi_{2}\left(u_{2}\right)\right)\right) \\
& =\left(\Gamma_{G_{1}}^{\pi\left(\psi_{2}\left(u_{2}\right)\right)+1}\left(\psi_{1}\left(u_{1}\right)\right), \psi_{2}\left(u_{2}\right)\right) \cup\left(\psi_{1}\left(u_{1}\right), \Gamma_{G_{2}}^{\pi\left(\psi_{1}\left(u_{1}\right)\right)+1}\left(\psi_{2}\left(u_{2}\right)\right)\right) \\
& =\left(\Gamma_{G_{1}}^{-}\left(\psi_{1}\left(u_{1}\right)\right), \psi_{2}\left(u_{2}\right)\right) \cup\left(\psi_{1}\left(u_{1}\right), \Gamma_{G_{2}}^{-}\left(\psi_{2}\left(u_{2}\right)\right)\right) \\
& =\Gamma_{H}^{-}\left(\psi_{1}\left(u_{1}\right), \psi_{2}\left(u_{2}\right)\right) \\
& =\Gamma_{H}^{-}\left(\Psi\left(u_{1}, u_{2}\right)\right) .
\end{aligned}
$$

This completes the proof.

\section{The Manhattan product and the Manhattan street networks}

Here we show the relation between the digraphs obtained by the Manhattan product and the Manhattan street networks.

Proposition 4.1. The Manhattan product of directed cycles with an even order $N_{i}, i=1,2, \ldots, n$, is a Manhattan street network. More precisely, if $G_{i}=C_{N_{i}}$, then

$$
C_{N_{1}} \# C_{N_{2}} \# \cdots \# C_{N_{n}}=M\left(N_{1}, N_{2}, \ldots, N_{n}\right) .
$$

Proof. For $i=1,2, \ldots, n$, each cycle $C_{N_{i}}$ has set of vertices $V_{i}=\mathbb{Z}_{N_{i}}$, and adjacencies $\Gamma^{+}\left(u_{i}\right)=$ $\left\{u_{i}+1 \bmod N_{i}\right\}$ and $\Gamma^{-}\left(u_{i}\right)=\left\{u_{i}-1 \bmod N_{i}\right\}$, such that $V_{i 0}$ and $V_{i 1}$ can be the sets of even and odd vertices, respectively. Thus, the set of vertices in the Manhattan product of directed cycles is $\mathbb{Z}_{N_{1}} \times \mathbb{Z}_{N_{2}} \times \cdots \times \mathbb{Z}_{N_{n}}$ and

$$
\left(u_{1}, \ldots, u_{i}, \ldots, u_{n}\right) \quad \rightarrow \quad\left(u_{1}, \ldots, v_{i}, \ldots, u_{n}\right), \quad i=1,2, \ldots, n
$$

where

- $v_{i}=u_{i}+1$ if and only if $\sum_{j \neq i} \pi\left(u_{j}\right)$ is even and, hence, $\sum_{j \neq i} u_{j}$ is also even,

- $v_{i}=u_{i}-1$ if and only if $\sum_{j \neq i} \pi\left(u_{j}\right)$ is odd and, hence, $\sum_{j \neq i} u_{j}$ is also odd, which corresponds to the definition of the Manhattan street network. 
As a consequence of the above proposition, we have the following result.

Corollary 4.1. The Manhattan product of two Manhattan street networks is a Manhattan street network. More precisely, if $M^{1}=M\left(N_{1}^{1}, N_{2}^{1}, \ldots, N_{n_{1}}^{1}\right)$ and $M^{2}=M\left(N_{1}^{2}, N_{2}^{2}, \ldots, N_{n_{2}}^{2}\right)$, then

$$
M^{1} \# M^{2}=M,
$$

where $M=M\left(N_{1}^{1}, \ldots, N_{n_{1}}^{1}, N_{1}^{2}, \ldots, N_{n_{2}}^{2}\right)$.

Proof. Both $M^{1}$ and $M^{2}$ are bipartite digraphs with vertex sets $V^{\alpha}=\mathbb{Z}_{N_{1}^{\alpha}} \times \mathbb{Z}_{N_{2}^{\alpha}} \times \cdots \times \mathbb{Z}_{N_{n_{\alpha}}^{\alpha}}$, $\alpha=1,2$; whereas $M^{1}$ \# $M^{2}$ has vertex set $V=V^{1} \times V^{2}$. Let $V(M)$ be the vertex set of $M$. Then, we claim that the natural mapping $\Psi: V \rightarrow V(M)$, defined by $\Psi\left(\boldsymbol{u}^{1}, \boldsymbol{u}^{2}\right)=$ $\left(u_{1}^{1}, \ldots, u_{n_{1}}^{1}, u_{1}^{2}, \ldots, u_{n_{2}}^{2}\right)$ is an isomorphism between the corresponding digraphs. In proving this, let $V_{0}^{\alpha}$ and $V_{1}^{\alpha}$ be the stable sets of $M^{\alpha}$ constituted, respectively, by the vertices $\boldsymbol{u}^{\alpha}=$ $\left(u_{1}^{\alpha}, \ldots, u_{n_{\alpha}}^{\alpha}\right)$, whose sum of components $\sum_{k=1}^{n_{\alpha}} u_{k}^{\alpha}$ is even or odd. With this convention, each vertex $\left(\boldsymbol{u}^{1}, \boldsymbol{u}^{2}\right)$ of the Manhattan product $M^{1} \# M^{2}$ is adjacent to the vertices $\left(\boldsymbol{v}^{1}, \boldsymbol{u}^{2}\right)$ and $\left(\boldsymbol{u}^{1}, \boldsymbol{v}^{2}\right)$ where, for the first ones,

- $\boldsymbol{v}^{1} \in \Gamma_{M^{1}}^{+}\left(\boldsymbol{u}^{1}\right)$ if $\pi\left(\boldsymbol{u}^{2}\right)$, and hence $\sum_{k=1}^{n_{2}} u_{k}^{2}$, is even;

- $\boldsymbol{v}^{1} \in \Gamma_{M^{1}}^{-}\left(\boldsymbol{u}^{1}\right)$ if $\pi\left(\boldsymbol{u}^{2}\right)$, and hence $\sum_{k=1}^{n_{2}} u_{k}^{2}$, is odd.

In the first case, for $i=1,2, \ldots, n_{1}$,

$$
\begin{aligned}
\left(\boldsymbol{v}^{1}, \boldsymbol{u}^{2}\right) & \stackrel{\Psi}{\mapsto}\left(u_{1}^{1}, \ldots, u_{i}^{1}+(-1)^{\sum_{j \neq i} u_{j}^{1}}, \ldots, u_{n_{1}}^{1}, u_{1}^{2}, \ldots, u_{n_{2}}^{2}\right) \\
& =\left(u_{1}^{1}, \ldots, u_{i}^{1}+(-1)^{\sum_{j \neq i} u_{j}^{1}+\sum_{k=1}^{n_{2}} u_{k}^{2}}, \ldots, u_{n_{1}}^{1}, u_{1}^{2}, \ldots, u_{n_{2}}^{2}\right) .
\end{aligned}
$$

Analogously, in the second case, for $i=1,2, \ldots, n_{2}$,

$$
\begin{aligned}
\left(\boldsymbol{v}^{1}, \boldsymbol{u}^{2}\right) & \stackrel{\Psi}{\mapsto}\left(u_{1}^{1}, \ldots, u_{i}^{1}-(-1)^{\sum_{j \neq i} u_{j}^{1}}, \ldots, u_{n_{1}}^{1}, u_{1}^{2}, \ldots, u_{n_{2}}^{2}\right) \\
& =\left(u_{1}^{1}, \ldots, u_{i}^{1}+(-1)^{\sum_{j \neq i} u_{j}^{1}+\sum_{k=1}^{n_{2}} u_{k}^{2}}, \ldots, u_{n_{1}}^{1}, u_{1}^{2}, \ldots, u_{n_{2}}^{2}\right) .
\end{aligned}
$$

Considering altogether both cases, we obtain (through all the $i$-arcs, $1 \leq i \leq n_{1}$ ) the vertices adjacent to $\Psi\left(\boldsymbol{u}^{1}, \boldsymbol{u}^{2}\right)=\left(u_{1}^{1}, \ldots, u_{n_{1}}^{1}, u_{1}^{2}, \ldots, u_{n_{2}}^{2}\right)$ in $M$. The adjacencies through the other $i$ arcs, $n_{1}+1 \leq i \leq n_{1}+n_{2}$ come from the vertices $\left(\boldsymbol{u}^{1}, \boldsymbol{v}^{2}\right)$.

The result of the previous proposition can also be seen as a corollary of Proposition 4.1 and the associative property. Indeed, we have

$$
\begin{aligned}
M^{1} \# M^{2} & =M\left(N_{1}^{1}, N_{2}^{1}, \ldots, N_{n_{1}}^{1}\right) \# M\left(N_{1}^{2}, N_{2}^{2}, \ldots, N_{n_{2}}^{2}\right) \\
& =\left(C_{N_{1}}^{1} \# C_{N_{2}}^{1} \# \cdots \# C_{N_{n_{1}}}^{1}\right) \#\left(C_{N_{1}}^{2} \# C_{N_{2}}^{2} \# \cdots \# C_{N_{n_{2}}}^{2}\right) \\
& =C_{N_{1}}^{1} \# C_{N_{2}}^{1} \# \cdots \# C_{N_{n_{1}}}^{1} \# C_{N_{1}}^{2} \# C_{N_{2}}^{2} \# \cdots \# C_{N_{n_{2}}}^{2} \\
& =M\left(N_{1}^{1}, N_{2}^{1}, \ldots, N_{n_{1}}^{1}, N_{1}^{2}, N_{2}^{2}, \ldots, N_{n_{2}}^{2}\right)=M .
\end{aligned}
$$




\section{The diameter}

In this section, we derive a precise bound of the Manhattan product of digraphs in terms of the unilateral diameters of its factors. In this context and by the way of comparison, notice that, according to the result in Theorem 2.1 $(d)$, the diameter $D$ of the Manhattan street network $M_{2}=$ $M\left(N_{1}, N_{2}\right)$ always satisfies the upper bound

$$
D \leq D_{1}^{*}+D_{2}^{*}+1
$$

where $D_{1}^{*}$ and $D_{2}^{*}$ are, respectively, the unilateral diameters of cycles $C_{N_{1}}$ and $C_{N_{2}}$. Now, we show that a very similar bound applies for the general case.

Theorem 5.1. Let $G_{1}$ and $G_{2}$ be digraphs with neither sources nor sinks (vertices with indegree or outdegree zero) and (finite) unilateral diameters $D_{1}^{*}$ and $D_{2}^{*}$, respectively. Then, the Manhattan product $H=G_{1} \# G_{2}$ has diameter $D$ satisfying

$$
D \leq D_{1}^{*}+D_{2}^{*}+2
$$

Proof. Let $\boldsymbol{u}=\left(u_{1}, u_{2}\right)$ and $\boldsymbol{v}=\left(v_{1}, v_{2}\right)$ be two generic vertices of $H$. We want to find a path from $\boldsymbol{u}$ to $\boldsymbol{v}$ with length not exceeding the claimed bound. With this aim, each vertex is denoted by $\left(\partial_{1}^{ \pm}, \partial_{2}^{ \pm}\right)$, where $\partial_{i}=\operatorname{dist}_{G_{i}}^{*}\left(u_{i}, v_{i}\right), i=1,2$, are referred to as their distance-components (with respect to $\boldsymbol{v}$ and, hence, such a destiny vertex is just $(0,0)$ ). The superscript + or - of $\partial_{1}$ indicates whether its parity is either the right one or not to follow the shortest path from $u_{2}$ to $v_{2}$ in $G_{2}$, and similarly for the superscript of $\partial_{2}$. Since the distances $\partial_{1}$ and $\partial_{2}$ can be either even or odd, and their parity can either agree $(+)$ or not $(-)$ with the shortest path of their mates, there are sixteen possible cases to be investigated which, by symmetry, are reduced to ten as follows:

(a1) $\left(2 x^{+}, 2 y^{+}\right)$;

(a2) $\left(2 x^{+}, 2 y^{-}\right) \equiv\left(2 x^{-}, 2 y^{+}\right)$

(a3) $\left(2 x^{-}, 2 y^{-}\right)$.

(b1) $\left(2 x^{+}, 2 y+1^{+}\right) \equiv\left(2 x+1^{+}, 2 y^{+}\right)$;

(b2) $\left(2 x^{+}, 2 y+1^{-}\right) \equiv\left(2 x+1^{-}, 2 y^{+}\right)$;

(b3) $\left(2 x^{-}, 2 y+1^{+}\right) \equiv\left(2 x+1^{+}, 2 y^{-}\right)$;

(b4) $\left(2 x^{-}, 2 y+1^{-}\right) \equiv\left(2 x+1^{-}, 2 y^{-}\right)$.

(c1) $\left(2 x+1^{+}, 2 y+1^{+}\right)$;

$(c 2)\left(2 x+1^{+}, 2 y+1^{-}\right) \equiv\left(2 x+1^{-}, 2 y+1^{+}\right)$; 


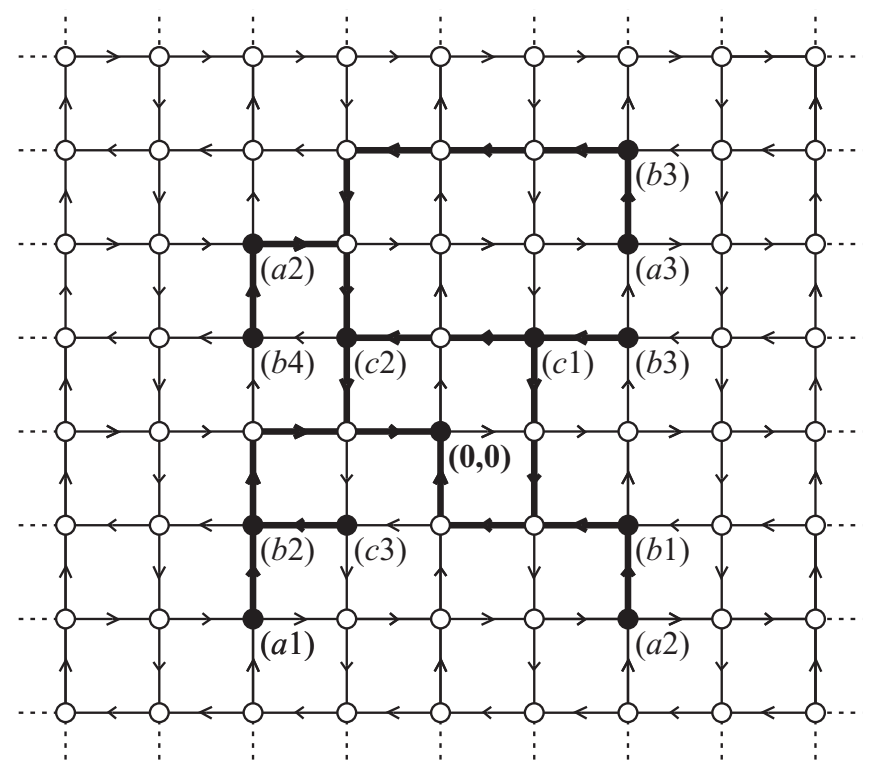

Figure 3. The different paths in the proof of Theorem 5.1

(c3) $\left(2 x+1^{-}, 2 y+1^{-}\right)$.

Then, a path from $\left(u_{1}, u_{2}\right)$ to $\left(v_{1}, v_{2}\right)$ corresponds to a path from $\left(\partial_{1}^{ \pm}, \partial_{2}^{ \pm}\right)$to $(0,0)$ as follows (see Fig. 3 when considering the Manhattan product of two cycles, that is, a Manhattan street network $M_{2}$ ):

(a1) $\left(2 x^{+}, 2 y^{+}\right) \stackrel{2 y}{\longrightarrow}\left(2 x, 0^{+}\right) \stackrel{2 x}{\longrightarrow}(0,0)$;

(a2) $\left(2 x^{+}, 2 y^{-}\right) \stackrel{2 y-1}{\longrightarrow}\left(2 x^{+}, 1^{+}\right) \stackrel{2 x}{\longrightarrow}\left(0^{+}, 1\right) \stackrel{1}{\longrightarrow}(0,0)$;

(a3) $\left(2 x^{-}, 2 y^{-}\right) \stackrel{1}{\longrightarrow}(b 1)\left(2 x^{+}, 2 z+1^{+}\right) \stackrel{2 x}{\longrightarrow} \cdots$; $\stackrel{1}{\longrightarrow}(b 3)\left(2 x^{-}, 2 z+1^{+}\right) \stackrel{2 x+1}{\longrightarrow} \cdots$;

(b1) $\left(2 x^{+}, 2 y+1^{+}\right) \stackrel{2 x}{\longrightarrow}\left(0^{+}, 2 y+1\right) \stackrel{2 y+1}{\longrightarrow}(0,0)$;

(b2) $\left(2 x+1^{-}, 2 y^{+}\right) \stackrel{2 x+1}{\longrightarrow}\left(0^{+}, 2 y\right) \stackrel{2 y}{\longrightarrow}(0,0) ;$

(b3) $\left(2 x^{-}, 2 y+1^{+}\right) \stackrel{2 x+1}{\longrightarrow}\left(1^{+}, 2 y+1^{-}\right) \stackrel{2 y+1}{\longrightarrow}\left(1,0^{+}\right) \stackrel{1}{\longrightarrow}(0,0)$;

(b4) $\left(2 x^{-}, 2 y+1^{-}\right) \stackrel{1}{\longrightarrow}(a 1)\left(2 x^{+}, 2 z^{+}\right) \stackrel{2 z}{\longrightarrow} \cdots$; $\stackrel{1}{\longrightarrow}(a 2)\left(2 x^{-}, 2 z^{+}\right) \stackrel{2 x-1}{\longrightarrow} \cdots$; 


$$
\begin{aligned}
& (c 1)\left(2 x+1^{+}, 2 y+1^{+}\right) \stackrel{2 y+2}{\longrightarrow}\left(2 x+1^{-}, 1^{+}\right) \stackrel{2 x+1}{\longrightarrow}\left(0^{+}, 1\right) \stackrel{1}{\longrightarrow}(0,0) ; \\
& (c 2)\left(2 x+1^{+}, 2 y+1^{-}\right) \stackrel{2 y+1}{\longrightarrow}\left(2 x+1,0^{+}\right) \stackrel{2 x+1}{\longrightarrow}(0,0) ; \\
& (c 3)\left(2 x+1^{-}, 2 y+1^{-}\right) \stackrel{1}{\longrightarrow}(b 1)\left(2 x+1^{+}, 2 z^{+}\right) \stackrel{2 z}{\longrightarrow} \cdots ; \\
& \stackrel{1}{\longrightarrow}(b 2)\left(2 x+1^{-}, 2 z^{+}\right) \stackrel{2 x+1}{\longrightarrow} \cdots .
\end{aligned}
$$

Note that, sometimes, we need to do some steps following a given (forced) orientation, so that the condition about having no vertices with null outdegree or indegree is necessary (and, clearly, also sufficient) for reaching the vertex desired. Then, the result follows by considering the maximum length of each of these paths taking into account that, if the diameter $D_{i}^{*}$ is even (respectively, odd) the unilateral distance between two vertices $u_{i}$ and $v_{i}$ of $G_{i}, i=1,2$, belonging to distinct (respectively, equal) independent sets is at most $D_{i}^{*}-1$. For instance, let us check in detail one particular case of (a3) where the upper bound can be reached, the other cases being analyzed similarly. With this aim, let us assume that $D_{1}^{*}$ and $D_{2}^{*}$ are even and consider the path

$$
\text { (a3) }\left(2 x^{-}, 2 y^{-}\right) \stackrel{1}{\longrightarrow}(b 3)\left(2 x^{-}, 2 z+1^{+}\right) \stackrel{2 x+1}{\longrightarrow}\left(1^{+}, 2 z+1^{-}\right) \stackrel{2 z+1}{\longrightarrow}\left(1,0^{+}\right) \stackrel{1}{\longrightarrow}(0,0) .
$$

First, the origin vertex $\boldsymbol{u}=\left(u_{1}, u_{2}\right)$ has distance-components $2 x^{-} \leq D_{1}^{*}$ and $2 y^{-} \leq D_{2}^{*}$. After 1 step, the vertex reached has second distance-component $2 z+1 \leq D_{2}^{*}$ (because of the parity of $\left.D_{2}^{*}\right)$. Then, we take $2 x+1 \leq D_{1}^{*}+1$ steps to reach a vertex with first component $v_{1}^{\prime} \in \Gamma_{G_{1}}^{+}\left(v_{1}\right)$ (and, hence, distance-component 1). Afterwards, $2 z+1 \leq D_{2}^{*}$ steps are done to reach the vertex with second components $v_{2}$, as required. Finally we take 1 extra step to reach the destiny vertex $\boldsymbol{v}=\left(v_{1}, v_{2}\right)$. Consequently, a total of $2 x+2 z+2 \leq D_{1}^{*}+D_{2}^{*}+2$ steps are required.

Notice that the above result implicitly gives a necessary and sufficient condition for the Manhattan product to be strongly connected (that is, with finite diameter). Moreover, as its proof is constructive, it provides a routing algorithm in $H$ from some given routing algorithms of its factors.

\section{Symmetries}

Now we study the symmetries of the digraphs obtained by the Manhattan product.

Proposition 6.1. Let $G_{i}$ be vertex-symmetric self-converse bipartite digraphs, $i=1,2, \ldots, n$. Then, the Manhattan product $H=G_{1} \# G_{2} \# \cdots \# G_{n}$ is a vertex-symmetric self-converse digraph.

Proof. As before, we only need to study the case $H=G_{1} \# G_{2}$, where $G_{i}=\left(V_{i}, A_{i}\right), V_{i}=$ $V_{i 0} \cup V_{i 1}, i=1,2$, are digraphs satisfying the hypotheses. Also, by Proposition 3.1 $(a)$, we already know that $H$ is isomorphic to its converse. Now, let us prove that there exists an automorphism $\Phi$ in $H$, which transforms any vertex $\left(u_{1}, u_{2}\right)$ into any vertex $\left(v_{1}, v_{2}\right)$. Depending on whether $u_{i}$ and $v_{i}, i=1,2$, are in the same or distinct partite sets $V_{i \alpha}, \alpha=0,1$, there are different cases to be considered: 
(i) If both pairs of components are in the same stable sets, $u_{1}, v_{1} \in V_{i \alpha}$ and $u_{2}, v_{2} \in V_{i \beta}$, we know that there exist automorphisms $\phi_{i}$ in $G_{i}, \phi_{i}\left(\Gamma_{G_{i}}^{ \pm}\left(w_{i}\right)\right)=\Gamma_{G_{i}}^{ \pm}\left(\phi_{i}\left(w_{i}\right)\right)$ for every $w_{i} \in V_{i}$, such that $\phi_{i}\left(u_{i}\right)=v_{i}, i=1,2$. Then, we define

$$
\Phi\left(w_{1}, w_{2}\right):=\left(\phi_{1}\left(w_{1}\right), \phi_{2}\left(w_{2}\right)\right) \quad \text { for every }\left(w_{1}, w_{2}\right) \in V(H),
$$

satisfying $\Phi\left(u_{1}, u_{2}\right)=\left(v_{1}, v_{2}\right)$ and, since $\pi\left(w_{i}\right)=\pi\left(\phi_{i}\left(w_{i}\right)\right)$ ( $\phi_{i}$ leaves invariant each partite set), we have:

$$
\begin{aligned}
\Phi\left(\Gamma_{H}^{+}\left(w_{1}, w_{2}\right)\right) & =\Phi\left(\left(\Gamma_{G_{1}}^{\pi\left(w_{2}\right)}\left(w_{1}\right), w_{2}\right)\right) \cup \Phi\left(\left(w_{1}, \Gamma_{G_{2}}^{\pi\left(w_{1}\right)}\left(w_{2}\right)\right)\right) \\
& =\left(\phi_{1}\left(\Gamma_{G_{1}}^{\pi\left(w_{2}\right)}\left(w_{1}\right)\right), \phi_{2}\left(w_{2}\right)\right) \cup\left(\phi_{1}\left(w_{1}\right), \phi_{2}\left(\Gamma_{G_{2}}^{\pi\left(w_{1}\right)}\left(w_{2}\right)\right)\right) \\
& =\left(\Gamma_{G_{1}}^{\pi\left(w_{2}\right)}\left(\phi_{1}\left(w_{1}\right)\right), \phi_{2}\left(w_{2}\right)\right) \cup\left(\phi_{1}\left(w_{1}\right), \Gamma_{G_{2}}^{\pi\left(w_{1}\right)}\left(\phi_{2}\left(w_{2}\right)\right)\right) \\
& =\left(\Gamma_{G_{1}}^{\pi\left(\phi_{2}\left(w_{2}\right)\right)}\left(\phi_{1}\left(w_{1}\right)\right), \phi_{2}\left(w_{2}\right)\right) \cup\left(\phi_{1}\left(w_{1}\right), \Gamma_{G_{2}}^{\pi\left(\phi_{1}\left(w_{1}\right)\right)}\left(\phi_{2}\left(w_{2}\right)\right)\right) \\
& =\Gamma_{H}^{+}\left(\phi_{1}\left(w_{1}\right), \phi_{2}\left(w_{2}\right)\right) \\
& =\Gamma_{H}^{+}\left(\Phi\left(w_{1}, w_{2}\right)\right),
\end{aligned}
$$

so that $\Phi$ is an automorphism of $H$.

(ii) If the pairs of components are in different stable sets, $u_{1} \in V_{i \alpha}, v_{1} \in V_{i \bar{\alpha}}$ and $u_{2} \in V_{i \beta}$, $v_{2} \in V_{i \bar{\beta}}$, and since $G_{i}, i=1,2$, are self-converse, there exist automorphisms $\psi_{i}$ from $G_{i}$ to $\bar{G}_{i}$, $\psi_{i}\left(\Gamma_{G_{i}}^{ \pm}\left(w_{i}\right)\right)=\Gamma_{G_{i}}^{\mp}\left(\psi_{i}\left(w_{i}\right)\right)$ for every $w_{i} \in V_{i}$, such that $\psi_{i}\left(u_{u}\right)=v_{i}$. We, then, define $\Phi$ as

$$
\Psi\left(w_{1}, w_{2}\right):=\left(\psi_{1}\left(w_{1}\right), \psi_{n}\left(w_{2}\right)\right) \quad \text { for every }\left(w_{1}, w_{2}\right) \in V(H),
$$

satisfying $\Phi\left(u_{1}, u_{2}\right)=\left(v_{1}, v_{2}\right)$ and, since $\pi\left(w_{i}\right)=0 \Longleftrightarrow \pi\left(\psi_{i}\left(w_{i}\right)\right)=1\left(\psi_{i}\right.$ interchanges the partite sets), we get

$$
\begin{aligned}
\Phi\left(\Gamma_{H}^{+}\left(w_{1}, w_{2}\right)\right) & =\Phi\left(\left(\Gamma_{G_{1}}^{\pi\left(w_{2}\right)}\left(w_{1}\right), w_{2}\right)\right) \cup \Phi\left(\left(w_{1}, \Gamma_{G_{2}}^{\pi\left(w_{1}\right)}\left(w_{2}\right)\right)\right) \\
& =\left(\psi_{1}\left(\Gamma_{G_{1}}^{\pi\left(w_{2}\right)}\left(w_{1}\right)\right), \psi_{2}\left(w_{2}\right)\right) \cup\left(\psi_{1}\left(w_{1}\right), \psi_{2}\left(\Gamma_{G_{2}}^{\pi\left(w_{1}\right)}\left(w_{2}\right)\right)\right) \\
& =\left(\Gamma_{G_{1}}^{\pi\left(w_{2}\right)+1}\left(\psi_{1}\left(w_{1}\right)\right), \psi_{2}\left(w_{2}\right)\right) \cup\left(\psi_{1}\left(w_{1}\right), \Gamma_{G_{2}}^{\pi\left(w_{1}\right)+1}\left(\psi_{2}\left(w_{2}\right)\right)\right) \\
& =\left(\Gamma_{G_{1}}^{\pi\left(\psi_{2}\left(w_{2}\right)\right)}\left(\psi_{1}\left(w_{1}\right)\right), \psi_{2}\left(w_{2}\right)\right) \cup\left(\psi_{1}\left(w_{1}\right), \Gamma_{G_{2}}^{\pi\left(\phi_{1}\left(w_{1}\right)\right)}\left(\psi_{2}\left(w_{2}\right)\right)\right) \\
& =\Gamma_{H}^{+}\left(\psi_{1}\left(w_{1}\right), \psi_{2}\left(w_{2}\right)\right) \\
& =\Gamma_{H}^{+}\left(\Phi\left(w_{1}, w_{2}\right)\right),
\end{aligned}
$$

which proves again that $\Phi$ is an automorphism of $H$.

(iii) Finally, in the case when one pair of components are in the same stable set and the other components are in distinct stable sets, say, $u_{1}, v_{1} \in V_{i \alpha}$ and $u_{2} \in V_{i \beta}, v_{2} \in V_{i \bar{\beta}}$, we can apply the same ideas of $(i)$ and $(i i)$. Namely, with the same notation as before, we prove that the mapping $\Phi$, defined as

$$
\Psi\left(w_{1}, w_{2}\right):=\left(\phi_{1}\left(w_{1}\right), \psi_{n}\left(w_{2}\right)\right) \quad \text { for every }\left(w_{1}, w_{2}\right) \in V(H),
$$

is the required automorphism of $H$. This completes the proof. 


\section{Cayley digraphs and the Manhattan product}

In this section, we investigate when the Manhattan product of Cayley digraphs is also a Cayley digraph. This generalizes the case studied in [4, 7] of Manhattan street networks, where the factors of the product are directed cycles (see Proposition 4.1), that is, Cayley digraphs of the cyclic groups. Because of the associative property of such a product, we only need to study the case of two factors.

Theorem 7.1. Let $G_{1}=\operatorname{Cay}\left(\Gamma_{1}, \Delta_{1}\right)$ be a bipartite Cayley digraph of the group $\Gamma_{1}$ with generating set $\Delta_{1}=\left\{a_{1}, \ldots, a_{p}\right\}$ and set of generating relations $R_{1}$, such that there exists a group automorphism $\psi_{1}$ satisfying $\psi_{1}\left(a_{i}\right)=a_{i}^{-1}$, for $i=1, \ldots$, p. Let $G_{2}=\operatorname{Cay}\left(\Gamma_{2}, \Delta_{2}\right)$ be a bipartite Cayley digraph of the group $\Gamma_{2}$ with generating set $\Delta_{2}=\left\{b_{1}, \ldots, b_{q}\right\}$ and set of generating relations $R_{2}$, such that there exists a group automorphism $\psi_{2}$ satisfying $\psi_{2}\left(b_{j}\right)=b_{j}^{-1}$, for $j=1, \ldots, q$. Then, the Manhattan product $H=G_{1} \# G_{2}$ is the Cayley digraph of the group

$$
\Gamma=\left\langle\alpha_{1}, \ldots, \alpha_{p}, \beta_{1}, \ldots, \beta_{q} \mid R_{1}^{\prime}, R_{2}^{\prime},\left(\alpha_{i} \beta_{j}\right)^{2}=\left(\alpha_{i} \beta_{j}^{-1}\right)^{2}=1, i \neq j\right\rangle,
$$

where $R_{1}^{\prime}$ is the same set of generating relations as $R_{1}$ changing $a_{i}$ by $\alpha_{i}$ (and similarly for $R_{2}^{\prime}$ ).

Proof. Since, for every $u_{1} \in \Gamma_{1}$ and $i=1, \ldots, p$,

$$
\psi_{1}\left(u_{1} a_{i}\right)=\psi\left(u_{1}\right) \psi\left(a_{i}\right)=\psi\left(u_{1}\right) a_{i}^{-1},
$$

then $\psi_{1}$ is an (involutive) isomorphism for $G_{1}$ to $\bar{G}_{1}$ preserving colors. The same statement holds for $\psi_{2}$ and $G_{2}$. Moreover, since $G_{1}, G_{2}$ are vertex-symmetric, Proposition 6.1 applies and $H$ is also vertex-symmetric. In fact, we will see that its automorphism group contains a regular subgroup. With this aim, note first that, by using the previous automorphisms, we have the following natural way of defining the adjacencies of $H$ (with 'colors' denoted by $\alpha_{i}, 1 \leq i \leq p$, and $\beta_{j}, 1 \leq j \leq q$ ):

$$
\begin{array}{ll}
\left(u_{1}, u_{2}\right) & \stackrel{\alpha_{i} \text {-arc }}{\longrightarrow}\left(u_{1}, u_{2}\right) * \alpha_{i}=\left(u_{1} \psi_{1}^{\pi\left(u_{2}\right)}\left(a_{i}\right), u_{2}\right), \\
\left(u_{1}, u_{2}\right) & \stackrel{\beta_{j} \text {-arc }}{\longrightarrow}\left(u_{1}, u_{2}\right) * \beta_{j}=\left(u_{1}, u_{2} \psi_{2}^{\pi\left(u_{1}\right)}\left(b_{j}\right)\right) .
\end{array}
$$

Let us now prove that the mappings $\phi_{1 i}, \phi_{2 j}$, for $1 \leq i \leq p$ and $1 \leq j \leq q$, defined by $\phi_{1 i}\left(u_{1}, u_{2}\right)=\left(a_{i} u_{1}, \psi_{2}\left(u_{2}\right)\right)$ and $\phi_{2 j}\left(u_{1}, u_{2}\right)=\left(\psi_{1}\left(u_{1}\right), b_{j} u_{2}\right)$ are all color-preserving isomorphisms of $H$. Indeed, for all $1 \leq i, j \leq p$, we have

$$
\begin{aligned}
& \phi_{1 i}\left(\left(u_{1}, u_{2}\right) * \alpha_{j}\right)=\phi_{1 i}\left(u_{1} \psi_{1}^{\pi\left(u_{2}\right)}\left(a_{j}\right), u_{2}\right)=\left(a_{i} u_{1} \psi_{1}^{\pi\left(u_{2}\right)}\left(a_{j}\right), \psi_{2}\left(u_{2}\right)\right) \\
& =\left(a_{i} u_{1} \psi_{1}^{\pi\left(\psi_{2}\left(u_{2}\right)\right)}\left(a_{j}\right), \psi_{2}\left(u_{2}\right)\right)=\left(a_{i} u_{1}, \psi_{2}\left(u_{2}\right)\right) * \alpha_{j}=\phi_{1 i}\left(u_{1}, u_{2}\right) * \alpha_{j},
\end{aligned}
$$

where we used that $\pi\left(u_{2}\right)=\pi\left(\psi_{2}\left(u_{2}\right)\right)$, because $u_{2}$ can be expressed as the product of the generators $b_{j}$ and $\pi\left(b_{j}\right)=\pi\left(\psi_{2}\left(b_{j}\right)\right)=\pi\left(b_{j}^{-1}\right)$, for all $1 \leq j \leq q$. Moreover, for all $1 \leq i \leq p$ and $1 \leq j \leq q$, we also have

$$
\begin{aligned}
& \phi_{1 i}\left(\left(u_{1}, u_{2}\right) * \beta_{j}\right)=\phi_{1 i}\left(u_{1}, u_{2} \psi_{2}^{\pi\left(u_{1}\right)}\left(b_{j}\right)\right)=\left(a_{i} u_{1}, \psi_{2}\left(u_{2}\right) \psi_{2}^{\pi\left(u_{1}\right)+1}\left(b_{j}\right)\right) \\
& =\left(a_{i} u_{1}, \psi_{2}\left(u_{2}\right) \psi_{2}^{\pi\left(a_{i} u_{1}\right)}\left(b_{j}\right)\right)=\left(a_{i} u_{1}, \psi_{2}\left(u_{2}\right)\right) * \beta_{j}=\phi_{1 i}\left(u_{1}, u_{2}\right) * \beta_{j},
\end{aligned}
$$


where we used that $u_{1}$ and $a_{i} u_{1}$ belong to different stable sets of $G_{1}$. Similarly, we obtain

$$
\begin{aligned}
\phi_{2 i}\left(\left(u_{1}, u_{2}\right) * \alpha_{j}\right) & =\phi_{2 i}\left(u_{1}, u_{2}\right) * \alpha_{j}, \quad 1 \leq i \leq q, 1 \leq j \leq p, \\
\phi_{2 i}\left(\left(u_{1}, u_{2}\right) * \beta_{j}\right) & =\phi_{2 i}\left(u_{1}, u_{2}\right) * \beta_{j}, \quad 1 \leq i, j \leq q .
\end{aligned}
$$

Now to see that the permutation group $\Gamma=\left\langle\phi_{1 i}, \phi_{2 j} \mid 1 \leq i \leq p, 1 \leq j \leq q\right\rangle$ acts transitively on $\Gamma_{1} \times \Gamma_{2}$, that is, on the vertex set of $H$, it is enough to show that any vertex $\left(u_{1}, u_{2}\right)$ can be mapped into vertex $\left(e_{1}, e_{2}\right)$ (where $e_{1}$ and $e_{2}$ stand for the identity elements of $\Gamma_{1}$ and $\Gamma_{2}$, respectively) since, as stated previously, $H$ is vertex-symmetric. To this end, as $\Delta_{1}$ is a generating set, $u_{1}^{-1}$ can be expressed in the form, say, $u_{1}^{-1}=a_{i_{1}} a_{i_{2}} \cdots a_{i_{r}}$. Then,

$$
\phi_{1 i_{1}} \phi_{1 i_{2}} \cdots \phi_{1 i_{r}}\left(u_{1}, u_{2}\right)=\left(a_{i_{1}} a_{i_{2}} \cdots a_{i_{r}} u_{1}, \psi_{2}^{r}\left(u_{2}\right)\right)=\left(e_{1}, \psi_{2}^{r}\left(u_{2}\right)\right)=\left(e_{1}, v_{2}\right),
$$

where $v_{2}=u_{2}^{(-1)^{r}}$ is either $u_{2}$ or $u_{2}^{-1}$ according to the parity of $r$. In any case, as $\Delta_{2}$ is also a generating set, the inverse of this element can be written as, say, $v_{2}^{-1}=b_{j_{1}} b_{j_{2}} \cdots b_{j_{s}}$. Then,

$$
\phi_{2 j_{1}} \phi_{2 j_{2}} \cdots \phi_{2 j_{s}}\left(e_{1}, v_{2}\right)=\left(\psi_{1}^{s}\left(e_{1}\right), e_{2}\right)=\left(e_{1}, e_{2}\right) .
$$

Thus, as claimed, the group $\Gamma$ is a regular subgroup of the automorphism group of $H$. According to Sabidussi's characterization [14], which states that a graph is a Cayley graph of a certain group if and only if its group of automorphisms admits a regular subgroup, the Manhattan product is the Cayley digraph of $\Gamma$ with generators $\alpha_{i} \equiv \phi_{1 i}$ and $\beta_{j} \equiv \phi_{2 j}, 1 \leq i \leq p, 1 \leq j \leq q$. Regarding the structure of $\Gamma$, let us check only one of the defining relations in (1), as the others can be proved similarly.

$$
\begin{aligned}
& \left(\phi_{1 i} \phi_{2 j}\right)^{2}\left(u_{1}, u_{2}\right)=\phi_{1 i} \phi_{2 j} \phi_{i 1} \phi_{2 j}\left(u_{1}, u_{2}\right)=\phi_{1 i} \phi_{2 j} \phi_{1 i}\left(\psi_{1}\left(u_{1}\right), b_{j} u_{2}\right) \\
& =\phi_{1 i} \phi_{2 j}\left(a_{i} \psi_{1}\left(u_{1}\right), b_{j}^{-1} \psi_{2}\left(u_{2}\right)\right)=\phi_{1 i}\left(a_{i}^{-1} \psi_{1}^{2}\left(u_{1}\right), \psi_{2}\left(u_{2}\right)\right) \\
& =\left(\psi_{1}^{2}\left(u_{1}\right), \psi_{2}^{2}\left(u_{2}\right)\right)=\left(u_{1}, u_{2}\right) .
\end{aligned}
$$

This completes the proof.

This result can be compared with the well-known following one (see White [16]): If $G_{1}$ and $G_{2}$ are, respectively, Cayley digraphs of groups $\Gamma_{1}=\left\langle a_{1}, \ldots, a_{p} \mid R_{1}\right\rangle$ and $\Gamma_{2}=\left\langle b_{1}, \ldots, b_{q} \mid R_{2}\right\rangle$, then its direct product $G_{1} \square G_{2}$ is the Cayley digraph of group

$$
\begin{aligned}
\Gamma & =\Gamma_{1} \times \Gamma_{2} \\
& =\left\langle\alpha_{1}, \ldots, \alpha_{p}, \beta_{1}, \ldots, \beta_{q} \mid R_{1}^{\prime}, R_{2}^{\prime}, \alpha_{i} \beta_{j}=\beta_{j} \alpha_{i}, 1 \leq i \leq p, 1 \leq j \leq q\right\rangle,
\end{aligned}
$$

with the same notation as before. As an example of direct product of Cayley digraphs, see Fig. 4, to be compared with the Manhattan product of the same digraphs shown in Fig. 1.

\section{An alternative definition}

The results of the preceding section, specifically the structure of the color-preserving automorphisms, suggest to study some alternative definitions of the Manhattan product of digraphs when they satisfy some conditions. More precisely, if each of the factors $G_{i}, i=1,2, \ldots, n$, of the Manhattan product has an involutive automorphism from $G_{i}$ to $\bar{G}_{i}$, we have the following result. 

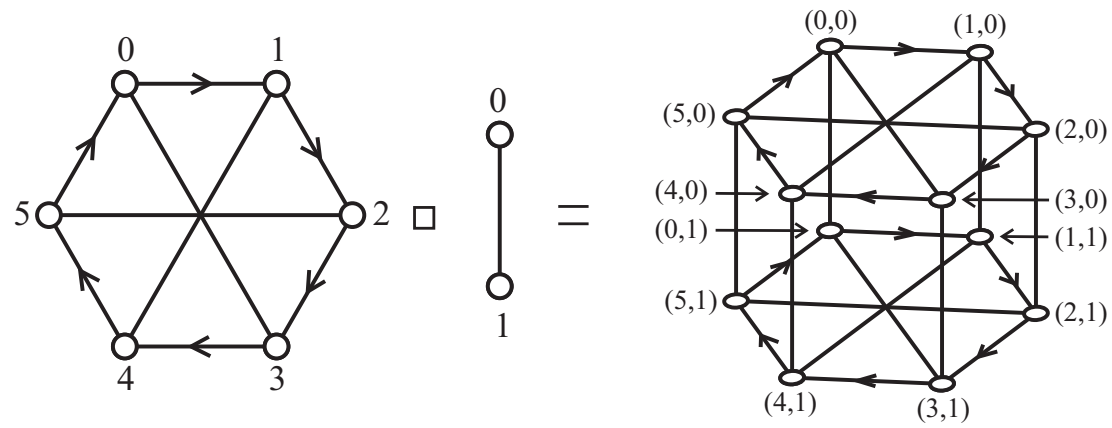

Figure 4. The direct product Cay $\left(\mathbb{Z}_{6},\{1,3\}\right) \square K_{2}^{*}$ (undirected lines stand for pairs of arcs with opposite directions).

Proposition 8.1. Let $\psi_{i}$ be an involutive automorphism from $G_{i}$ to $\bar{G}_{i}$, for $i=1,2, \ldots, n$. Then, the Manhattan product $H=G_{1} \# G_{2} \# \ldots$ \# $G_{n}$ is the digraph with vertex set $V\left(M_{n}\right)=$ $\mathbb{Z}_{N_{1}} \times \mathbb{Z}_{N_{2}} \times \cdots \times \mathbb{Z}_{N_{n}}$ and the following adjacencies:

$$
\left(u_{1}, u_{2}, \ldots, u_{i}, \ldots, u_{n}\right) \rightsquigarrow\left(\psi_{1}\left(u_{1}\right), \psi_{2}\left(u_{2}\right), \ldots, v_{i}, \ldots, \psi_{n}\left(u_{n}\right)\right), i=1,2, \ldots, n,
$$

where $v_{i} \in \Gamma^{+}\left(u_{i}\right)$.

Proof. We write, respectively, the adjacencies of the first definition and the alternative one as

$$
\begin{gathered}
\left(u_{1}, \ldots, u_{i}, \ldots, u_{n}\right) \rightarrow\left(u_{1}, \ldots, \Gamma^{\sum_{j \neq i} \pi\left(u_{j}\right)}\left(u_{i}\right), \ldots, u_{n}\right), i=1,2, \ldots, n, \\
\left(u_{1}, \ldots, u_{i}, \ldots, u_{n}\right) \rightsquigarrow\left(\psi_{1}\left(u_{1}\right), \ldots, \Gamma^{+}\left(u_{i}\right), \ldots, \psi_{n}\left(u_{n}\right)\right), i=1,2, \ldots, n .
\end{gathered}
$$

The isomorphism from the digraph obtained by the first definition to the digraph obtained by the alternative one is

$$
\begin{aligned}
& \Phi\left(u_{1}, \ldots, u_{i}, \ldots, u_{n}\right)= \\
& \left(\psi_{1}^{\sum_{j \neq 1}^{\sum_{j} \pi\left(u_{j}\right)}}\left(u_{1}\right), \ldots, \psi_{i}^{\sum_{j \neq i} \pi\left(u_{j}\right)}\left(u_{i}\right), \ldots, \psi_{n}^{\sum_{j \neq n} \pi\left(u_{j}\right)}\left(u_{n}\right)\right) .
\end{aligned}
$$

Indeed, let us see that this mapping preserves the adjacencies. First, by (2), we have

$$
\begin{aligned}
& \Phi\left(\Gamma^{+}\left(u_{1}, \ldots, u_{i}, \ldots, u_{n}\right)\right)= \\
& \left(\psi_{1}^{\sum_{j \neq 1} \pi\left(u_{j}\right)+1}\left(u_{1}\right), \ldots, \psi_{i}^{\sum_{j \neq i} \pi\left(u_{j}\right)}\left(\Gamma^{\sum_{j \neq i} \pi\left(u_{j}\right)}\left(u_{i}\right)\right), \ldots, \psi_{n}^{\sum_{j \neq n} \pi\left(u_{j}\right)+1}\left(u_{n}\right)\right) .
\end{aligned}
$$

Whereas, by (3), we have

$$
\begin{aligned}
& \Gamma^{+}\left(\Phi\left(u_{1}, \ldots, u_{i}, \ldots, u_{n}\right)\right)= \\
& \left(\psi_{1}^{\sum_{j \neq 1} \pi\left(u_{j}\right)+1}\left(u_{1}\right), \ldots, \Gamma^{+}\left(\psi_{i}^{\sum_{j \neq i} \pi\left(u_{j}\right)}\left(u_{i}\right)\right), \ldots, \psi_{n}^{\sum_{j \neq n} \pi\left(u_{j}\right)+1}\left(u_{n}\right)\right) .
\end{aligned}
$$

To check that, for every $i=1,2, \ldots, n$, the $i$-th entry in (4) and (5) represents the same set, we distinguish two cases: 
- If $\sum_{j \neq i} \pi\left(u_{j}\right)=\sigma$ is an even number, then $\psi_{i}^{\sigma}=I d$ (as $\psi_{i}$ is involutive) and $\operatorname{Id}\left(\Gamma^{+}\left(u_{i}\right)\right)=$ $\Gamma^{+}\left(\operatorname{Id}\left(u_{i}\right)\right)$.

- If $\sum_{j \neq i} \pi\left(u_{j}\right)=\sigma$ is an odd number, then $\psi_{i}^{\sigma}=\psi_{i}$ and $\psi_{i}\left(\Gamma^{-}\left(u_{i}\right)\right)=\Gamma^{+}\left(\psi_{i}\left(u_{i}\right)\right)$ (as $\psi_{i}$ is an automorphism from $G_{i}$ to $\bar{G}_{i}$ ).

In the case of the Manhattan street network $M_{n}, G_{i}=C_{i}$ (Proposition 4.1), $i=1,2, \ldots, n$. Then, a simple way of choosing the involutive automorphisms is $\psi_{i}\left(u_{i}\right)=-u_{i} \bmod N_{i}$ (in fact, it is readily checked that any isomorphism from $C_{i}$ to $\bar{C}_{i}$ is involutive). This gives the following definition of $M_{n}$ [4, 7]: The Manhattan street network $M_{n}=M_{n}\left(M_{1}, \ldots, M_{n}\right)$ is the digraph with vertex set $\mathbb{Z}_{N_{1}} \times \cdots \times \mathbb{Z}_{N_{n}}$ and adjacencies

$$
\left(u_{1}, \ldots, u_{i}, \ldots, u_{n}\right) \rightsquigarrow\left(-u_{1}, \ldots, u_{i}+1, \ldots,-u_{n}\right), \quad i=1,2, \ldots, n .
$$

\section{Hamiltonian Cycles}

Next, we give a result on the Hamiltonicity of the Manhattan product of two digraphs with Hamiltonian paths, as a generalization of a theorem in $[4,7]$ about the Hamiltonicity of the Manhattan street network.

Theorem 9.1. If $G_{1}$ and $G_{2}$ have both a Hamiltonian path, then their Manhattan product $H=$ $G_{1} \# G_{2}$ is Hamiltonian.

Proof. We construct a Hamiltonian cycle in $H$, from the Hamiltonian paths in $G_{1}$ and $G_{2}$, say $1 \rightarrow 2 \rightarrow \cdots \rightarrow N_{1}$ and $1^{\prime} \rightarrow 2^{\prime} \rightarrow \cdots \rightarrow N_{2}$, respectively. With this aim, we appropriately joint $N_{2}$ Hamiltonian paths (some of them without an arc) of $N_{2}$ subdigraphs isomorphic to $G_{1}$ or $\bar{G}_{1}$ (see Proposition 3.1(c)). Such paths are joined by using three copies of Hamiltonian paths (two of them with alternative arcs removed) of subdigraphs isomorphic to $G_{2}$ or $\bar{G}_{2}$. See the self-explanatory Fig. 5. 


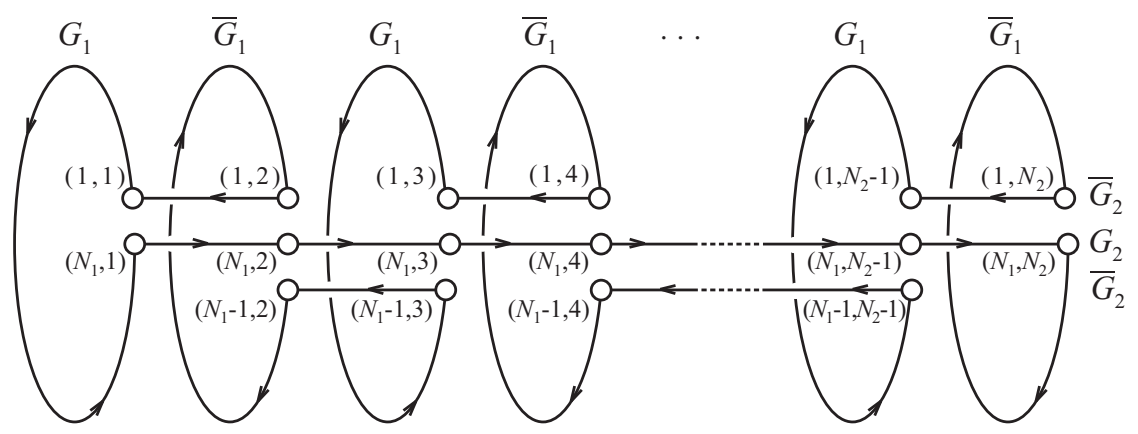

Figure 5. A Hamiltonian cycle in the Manhattan product $G_{1} \# G_{2}$.

\section{Acknowledgement}

This research was supported by the Ministry of Science and Innovation (Spain) and the European Regional Development Fund under project MTM2011-28800-C02-01-1 and by the Catalan Research Council under project 2009SGR1387.

[1] J. Bang-Jensen and G. Gutin, Digraphs: Theory, algorithms and applications, Springer Monographs in Mathematics, Springer, London, 2003.

[2] G. Chartrand and L. Lesniak, Graphs \& Digraph, Chapman and Hall, London, 1996.

[3] T. Y. Chung and D. P. Agrawal, Design and analysis of multidimensional Manhattan Street Networks, IEEE Trans. Commun. 41 (1993), 295-298.

[4] F. Comellas, C. Dalfó and M. A. Fiol, The multidimensional Manhattan networks, SIAM J. Discrete Math. 22 (2008), 1428-1447.

[5] F. Comellas, C. Dalfó and M. A. Fiol, A new operation on digraphs: The Manhattan product, VI Jornadas de Matemática Discreta y Algorítmica, pp. 279-286, Edicions i Publicacions de la UdL, Universitat de Lleida, 2008.

[6] F. Comellas, C. Dalfó, M.A. Fiol and M. Mitjana, The spectra of Manhattan street networks, Linear Algebra Appl. 429 (2008), no. 7, 1823-1839.

[7] C. Dalfó, F. Comellas and M.A. Fiol, The multidimensional Manhattan street networks, Electron. Notes Discrete Math. 29 (2007), 383-387.

[8] J. Gómez, E.A. Canale and X. Muñoz, On the unilateral $\left(\Delta, D^{*}\right)$-problem, Networks 36 (2000), no. 3, 164-171.

[9] J. Gómez, E.A. Canale and X. Muñoz, Unilaterally connected large digraphs and generalized cycles, Networks 42 (2003), no. 4, 181-188. 
[10] W. Imrich and S. Klavzar, Product graphs: structure and recognition, John Wiley \& sons, New York, 2000.

[11] B. Khasnabish, Topological properties of Manhattan Street Networks, Electronics Lett. 25 (1989), 1388-1389.

[12] N.F. Maxemchuk, Routing in the Manhattan Street Network, IEEE Trans. Commun. 35 (1987) 503-512.

[13] P. Morillo, M. A. Fiol and J. Fàbrega, The diameter of directed graphs associated to plane tessellations, Ars Comb. 20A (1985), 17-27.

[14] G. Sabudussi, On a class of fixed-point-free graphs, Proc. Amer. Math. Soc. 9 (1958), 800804.

[15] E. A. Varvarigos, Optimal communication algorithms for Manhattan Street Networks, Discrete Appl. Math. 83 (1998), 303-326.

[16] A.T. White, Graphs, groups and surfaces, North-Holland, Amsterdam, 1984. 